D. J. Dewsnap, Southeastern Oklahoma State University, P. O. Box 4194, Durant, OK 74701-0609. e-mail: ddewsnap@sosu.edu

P. Fischer, Department of Mathematics and Statistics, University of Guelph, Guelph, ON N1G 2W1, Canada. e-mail: pfischer@uoguelph.ca

\title{
NON-UNIQUENESS OF COMPOSITION SQUARE ROOTS
}

\begin{abstract}
In response to a question posed by O.E. Lanford III, it is shown that for each $\mu>0$ there is a differentiable and non-linearizable interval map $g$ with non-vanishing derivative defined on a neighborhood of a fixed point 0 with $g^{\prime}(0)=\mu$ such that $g$ has infinitely many differentiable and non-linearizable orientation-reversing composition square roots with non-vanishing first derivatives on a neighborhood of 0 .
\end{abstract}

\section{Introduction}

Throughout this paper, $f^{k}$ will denote the $k^{\prime}$ th iterate of $f$ and $I$ shall denote an interval of the reals of finite positive length. A neighborhood $J$ of $0 \in I$ shall refer to a subinterval of $I$ having 0 as an interior point with corresponding leftneighborhood defined by $J^{-}(0)=J \cap\{x \in I \mid x \leq 0\}$ and right-neighborhood $J^{+}(0)$ similarly defined. The Koenigs' sequence $\left\{\phi_{k}(f)\right\}$ associated with an interval map $f$ defined on a neighborhood of a fixed point 0 with $f^{\prime}(0)=\lambda$ where $0<|\lambda|<1$ is defined by $\phi_{k}(f)(x)=f^{k}(x) / \lambda^{k}$. If the Koenigs' sequence of $f$ converges pointwise to a limit $\phi$ on a neighborhood $I$ of 0 , then $\phi$ satisfies the Schröder equation $\phi(f(x))=\lambda \phi(x)$ on $I$. We will use the following two results from [1].

Theorem 1.1. Let $f$ be defined on a neighborhood of a fixed point 0 with $f^{\prime}(0)=\lambda$ where $-1<\lambda<0$. If either $f \circ f(x) \leq \lambda^{2} x$ or $f \circ f(x) \geq \lambda^{2} x$ on a neighborhood of 0 , then Koenigs' sequence converges uniformly to a limit $\phi$ on a neighborhood of 0 with $\phi(0)=0$ and $\phi^{\prime}(0)=1$.

Key Words: composition square root, Koenigs' sequence, linearizable, Schröder equation

Mathematical Reviews subject classification: 37E05, 58F03

Received by the editors February 16, 2000

* Work supported in part by the NSERC of Canada under grant A-8421. 
The following proposition identifies functions satisfying the conditions of Theorem 1.1.

Proposition 1.2. Let $p(x)=x(\alpha x+\lambda)$ and $q(x)=x(\beta x+\lambda)$ for each $x \in \boldsymbol{R}$ with $-1<\lambda<0$ and $0<\alpha<\beta<-\alpha / \lambda$. If $f$ is a function satisfying $p(x) \leq f(x) \leq q(x)$ on a neighborhood of 0 , then $f^{\prime}(0)=\lambda$ and $f \circ f(x) \leq \lambda^{2} x$ on a neighborhood of 0 .

Let $f$ be defined on a neighborhood of a fixed point 0 with $f^{\prime}(0)=\lambda$ where $\lambda$ is a real number. The interval map $f$ is said to be linearizable if there exists an invertible function $\phi$ with $\phi(0)=0, \phi^{\prime}(0)=1$, and satisfying $\phi \circ f \circ \phi^{-1}=\lambda x$ on a neighborhood of 0 . Such a $\phi$ will be referred to as a conjugator to $f$. It follows that if $f$ is linearizable, then it is invertible on a neighborhood of 0 unless $\lambda=0$. As well, $\phi$ satisfies the Schröder equation for $f$ on a neighborhood of 0 .

Consider an interval map $g$ defined on a neighborhood of a fixed point 0 with $g^{\prime}(0)=\lambda^{2}$ where $\lambda<0$. A composition square root of $g$ is a function $f$ satisfying $f^{2}=g$ on some neighborhood $I$ of the fixed point 0 . We refer to $f$ as being an orientation-reversing composition square root of $g$ if $f\left(I^{-}(0)\right) \subset$ $[0, \infty)$ and $f\left(I^{+}(0)\right) \subset(-\infty, 0]$. If $f$ is differentiable at the fixed point 0 , then $f^{\prime}(0)=\lambda$. A problem will be said to have a unique solution if all solutions of the problem belong to the same germ of functions. The equivalence relation which determines the germs defines two functions to be equivalent if there exists a neighborhood of 0 on which they coincide. We state the following result without proof.

Theorem 1.3. (O.E. Lanford III) Let $g$ be defined on a neighborhood of a fixed point 0 with $g^{\prime}(0)=\lambda^{2}$ where $-1 \neq \lambda<0$. If $g$ is linearizable, then there exists a unique orientation-reversing composition square root $f$ with $f^{\prime}(0)=\lambda$.

For a map $g$ defined on a neighborhood of a fixed point 0 with $g^{\prime}(0)=$ $\lambda^{2}=1$, then $g$ is linearizable if and only if $g(x)=x$ on a neighborhood of 0 . Indeed, if $\phi$ is a conjugator to $g$, then it must satisfy the Schröder equation $\phi \circ g(x)=\phi(x)$ on a neighborhood of 0 . Since both $\phi$ and $g$ are invertible on a neighborhood of 0 and continuous at 0 , it follows that $g$ must be the identity on a neighborhood of 0 . Clearly, linearization of $g(x)=x$ can be achieved by many conjugators. Any orientation-reversing composition square root $f$ of $g(x)=x$ which is differentiable at 0 must satisfy the equation $f^{2}(x)=x$ with $f(0)=0$ and $f^{\prime}(0)=\lambda=-1$. If $f$ is strictly decreasing and continuous with $f=f^{-1}, f(0)=0$, and $f^{\prime}(0)=-1$, then $f$ is an orientationreversing composition square root of $g$ which is also differentiable at the fixed point. Thus, when $\lambda=-1$ there are infinitely many strictly decreasing and 
continuous (in fact, analytic) orientation-reversing composition square roots of a linearizable mapping that are differentiable at the fixed point.

For a continuous, strictly increasing, and non-linearizable $g$ defined on a neighborhood of a fixed point 0 with $0<g^{\prime}(0)<1$, the question of uniqueness of a strictly decreasing and continuous orientation-reversing composition square root that is differentiable at the fixed point was posed by O.E. Lanford III. We will show that the answer to this question is in the negative.

\section{Non-Uniqueness Example}

We begin with an example of a differentiable and non-linearizable interval map $g$ with non-vanishing derivative defined on a neighborhood of a fixed point 0 with $0<g^{\prime}(0)<1$. This function will be shown to have infinitely many differentiable orientation-reversing composition square roots with nonvanishing first derivatives on a neighborhood of 0 .

Example 2.1. Let $p$ and $q$ be quadratic polynomials satisfying the conditions of Proposition 1.2 on a neighborhood $J$ of the fixed point 0 with $p$ and $q$ both strictly decreasing on $J$ and $q\left(J^{-}(0)\right) \subseteq J^{+}(0)$. Let $r$ be a quadratic polynomial satisfying $p(x)<r(x)<q(x)$ for each $x \in J \backslash\{0\}$. Choose $u \in J^{-}(0) \backslash\{0\}$ sufficiently close to 0 so that the sequence of points $\left\{w_{k}\right\}_{k=0}^{\infty}$ satisfying $q\left(w_{2 k}\right)=r\left(r^{2 k}(u)\right)$ and $p\left(w_{2 k+1}\right)=r\left(r^{2 k+1}(u)\right)$ also satisfies $w_{2 k} \in$ $\left(r^{2 k}(u), r^{2 k+2}(u)\right)$ and $w_{2 k+1} \in\left(r^{2 k+3}(u), r^{2 k+1}(u)\right)$ for each $k \geq 0$. The sequence $\left\{\epsilon_{k}\right\}_{k=0}^{\infty}$ is defined recursively by choosing $0<\epsilon_{0}<w_{0}-u$ and $0<(-1)^{k} \epsilon_{k}<\min \left\{\left|r^{k}(u)-r\left(r^{k-1}(u)+\epsilon_{k-1}\right)\right|,\left|r^{k}(u)-w_{k}\right|\right\}$ for each $k \geq 1$, where the $\epsilon_{k}$ 's are additionally chosen so that $\lim _{k \rightarrow \infty} \epsilon_{k} / \lambda^{k}=0$. We define a differentiable and non-linearizable function $f$ satisfying $f^{\prime}(x)<0$ and $p(x) \leq$ $f(x) \leq q(x)$ on the interval $[u, r(u)]$ in the following manner:

Let $f(0)=0$ and $f^{k}(u)=r^{k}(u)$ for each $k \geq 0$. Let $v=u+\epsilon_{0}$ and $f^{k}(v)=$ $f^{k}(u)+\epsilon_{k}$ for each $k \geq 1$. The choice of $\left\{\epsilon_{k}\right\}_{k=0}^{\infty}$ ensures that $r\left(r^{2 k}(u)+\epsilon_{2 k}\right)=$ $r\left(f^{2 k}(v)\right)<f\left(f^{2 k}(v)\right)<r\left(r^{2 k}(u)\right)=q\left(w_{2 k}\right)<q\left(f^{2 k}(v)\right)$ and $p\left(f^{2 k+1}(v)\right)<$ $p\left(w_{2 k+1}\right)=r\left(r^{2 k+1}(u)\right)<f\left(f^{2 k+1}(v)\right)<r\left(f^{2 k+1}(v)\right)=r\left(r^{2 k+1}(u)+\epsilon_{2 k+1}\right)$. Define $f$ on the remaining points in $[u, r(u)]$ so that $f$ is smooth everywhere except at the origin with $f^{\prime}(x)<0$ and $p(x)<f(x)<q(x)$ on $[u, r(u)] \backslash\{0\}$. As a result of Proposition 1.2, $f$ is differentiable at 0 with $f^{\prime}(0)=\lambda$ and $f \circ f(x) \leq \lambda^{2} x$ on a neighborhood of 0 . It follows from Theorem 1.1 that the Koenigs' sequences for both $f$ and $g=f^{2}$ converge uniformly on a subinterval $I \subseteq[u, r(u)]$ containing 0 to limit $\phi$ with $\phi(0)=0$ and $\phi^{\prime}(0)=1$. Since $f$ is continuous and strictly decreasing on $I$, then $\phi$ is continuous and increasing on $I$. Also, since $f^{k}(v)=f^{k}(u)+\epsilon_{k}$ with $\lim _{k \rightarrow \infty} \epsilon_{k} / \lambda^{k}=0$, it follows that 
$\phi\left(f^{k}(u)\right)=\phi\left(f^{k}(v)\right)$ for each $k \geq 0$. Thus, $\phi$ is constant on each of the subintervals $\left[f^{2 k}(u), f^{2 k}(v)\right]$ and $\left[f^{2 k+1}(v), f^{2 k+1}(u)\right]$ for each $k \geq 0$.

In conclusion, $g$ is smooth everywhere on $I$ except at 0 and $g^{\prime}(x)>0$ everywhere on $I$ with $g^{\prime}(0)=\lambda^{2}$ where $-1<\lambda<0$. The Koenigs' sequence for $g$ converges uniformly on $I$ to limit $\phi$, where $\phi$ is a continuous and increasing function on $I$ with $\phi(0)=0$ and $\phi^{\prime}(0)=1$. Since $\phi$ is non-invertible on any neighborhood of 0 , it follows that $g$ is non-linearizable at 0 .

We will need the following lemma.

Lemma 2.2. (O.E. Lanford III) Let $g$ be strictly increasing and continuous on the interval $[a, b]$ where $a<0<b$ with $g(0)=0, g(x)>x$ for $a \leq x<0$, and $g(x)<x$ for $0<x \leq b$. Let $f$ be strictly decreasing and continuous on the interval $[a, g(a)]$ with $f(a) \leq b$ and $f(g(a))>0$. Then $f$ can be extended to the interval $[a, f(a)]$ as a strictly decreasing and continuous function such that $f^{2}=g$ if and only if $g(f(a))=f(g(a))$. If these previous conditions hold, then the extension is unique.

We will now show that the map $g$ of Example 2.1 has infinitely many strictly decreasing and differentiable orientation-reversing composition square roots.

Example 2.3. Consider $g=f^{2}$ of Example 2.1. We obtain a differentiable function $h \neq f$ with $h^{2}=g, h^{\prime}(0)=\lambda$, and $h^{\prime}(x)<0$ on $I$ in the following manner :

We have $I \subseteq[u, f(u)]$. By replacing $u$ and $v$ with $f^{2 n}(u)$ and $f^{2 n}(v)$ for sufficiently large $n$ we may assume that $I=[u, f(u)]$ where $u<v<$ $f^{2}(u)$. We define $h$ so that $h(u)=f(u), h(x)=f(x)$ for $x \in\left[v, f^{2}(u)\right]$, $h^{\prime}(x)<0$ and continuous on $[u, v]$, and $h(x)>f(x)$ for each $x \in(u, v)$. It is clear that such an $h$ exists and satisfies the condition $h(g(u))=g(h(u))$. In view of Lemma $2.2, h$ extends uniquely to a continuous strictly decreasing function satisfying $h^{2}=g$ on $I$, where the extended function is also denoted by $h$. Furthermore, it follows that $h(x)=f(x)$ everywhere on $I$ except on the subintervals $\left(f^{2 k}(u), f^{2 k}(v)\right)$ and $\left(f^{2 k+1}(v), f^{2 k+1}(u)\right)$ for each $k \geq 0$. On the other hand, one can show by induction that if $x \in(u, v)$ then $h\left(f^{n}(x)\right)>$ $f\left(f^{n}(x)\right)$ for all positive integers $n$. Only a simple case will be considered here, which is already sufficient to conclude that $h \neq f$ in the sense of germs. For $x \in(u, v)$ we have $h\left(f^{2 n}(x)\right)=g^{n}(h(x))>g^{n}(f(x))=f\left(f^{2 n}(x)\right)$, since $g^{n}$ is strictly increasing. Furthermore, since $h^{\prime}(h(x))=g^{\prime}(x) / h^{\prime}(x)$, it follows by induction that $h^{\prime}(x)<0$ and $h$ is smooth everywhere on $I \backslash\{0\}$. To show that $h^{\prime}(0)=\lambda$, it is sufficient to show that $\lim _{k \rightarrow \infty} h\left(h^{k}(x)\right) / h^{k}(x)=\lambda$ uniformly on $(u, v)$. We prove that $\lim _{k \rightarrow \infty} h\left(h^{2 k}(x)\right) / h^{2 k}(x)=\lambda$ uniformly on $(u, v)$. 
We have

$$
\frac{f\left(f^{2 k}(u)\right)}{f^{2 k}(u)+\epsilon_{2 k}}=\frac{f\left(f^{2 k}(u)\right)}{f^{2 k}(v)}=\frac{h\left(h^{2 k}(u)\right)}{h^{2 k}(v)}<\frac{h\left(h^{2 k}(x)\right)}{h^{2 k}(x)}<\frac{f\left(h^{2 k}(x)\right)}{h^{2 k}(x)}
$$

for every $x \in(u, v)$. Since $f$ satisfies the conditions of Theorem 1.1, then

$$
\frac{f^{2 k}(u)+\epsilon_{2 k}}{f\left(f^{2 k}(u)\right)}=\frac{f^{2 k}(u)}{f\left(f^{2 k}(u)\right)}+\frac{\epsilon_{2 k} / \lambda^{2 k+1}}{f^{2 k+1}(u) / \lambda^{2 k+1}} \stackrel{k}{\longrightarrow} \frac{1}{\lambda},
$$

where $\lim _{k \rightarrow \infty} f^{2 k+1}(u) / \lambda^{2 k+1}=\phi(u)<0$ and $\lim _{k \rightarrow \infty} \epsilon_{2 k} / \lambda^{2 k+1}=0$. The proof that $\lim _{k \rightarrow \infty} h\left(h^{2 k+1}(x)\right) / h^{2 k+1}(x)=\lambda$ uniformly on $(u, v)$ is similar. This completes the proof.

In the previous example $f, h$, and $g$ are differentiable and invertible functions with non-vanishing derivatives on $I$. Thus, $f^{-1}$ and $h^{-1}$ are differentiable orientation-reversing composition square roots of $g^{-1}$ on $g(I)$. Combining this with the case $g^{\prime}(0)=\lambda^{2}=1$ where $\lambda=-1$ discussed in the Introduction, we obtain the following result.

Theorem 2.4. For each $\mu>0$ there is a differentiable $g$ with non-vanishing derivative defined on a neighborhood of a fixed point 0 with $g^{\prime}(0)=\mu$ such that $g$ has infinitely many differentiable orientation-reversing composition square roots with non-vanishing first derivatives on a neighborhood of 0 .

\section{Acknowledgements}

The authors wish to thank Professor Oscar Lanford III for his comments and suggestions, which have considerably improved this paper.

\section{References}

[1] D.J. Dewsnap and P. Fischer, Interval Maps and Koenigs' Sequences, Real Anal. Exchange, 25 (1999/2000), no. 1, 205-221. 
D. J. Dewsnap and P. Fischer 